\title{
NOISE INTO PLAY. DISONANCIAS E INDETERMINACIÓN DE LO SONORO EN LO LÚDICO
}

\author{
Moisés Mañas Carbonell \\ Marina Pastor Aguilar \\ Universidad Politécnica de Valencia. Dpto. Escultura. \\ Máster en Artes Visuales y Multimedia
}

\section{Resumen}

Este artículo pretende plantear un estudio relacional entre el concepto de ruido sonoro y aspectos indeterministas de la lúdica interactiva experimental, centrándose en los Survival Horror Games. Partiendo de la capacidad del sonido para desbordar la interface pantalla y conectar con el espacio real en el que se encuentra el jugador, se establece una conexión con el concepto de ética en los videojuegos desarrollado por Miguel Sicart. La propuesta atiende tanto a los aspectos experimentales de la producción sonora que se encuentran en el diseño del juego, y su relación con el campo del arte sonoro, como a las posibilidades de abrir el campo de lo político y la acción común desde de la experiencia de los jugadores, a partir de un movimiento semántico en el concepto de terror que puede transformar la experiencia del mismo en el espacio real.

Palabras clave: ARTE SONORO; INTERFACES LÚDICAS; GAMEART; VIDEOJUEGOS; INTERACCIÓN

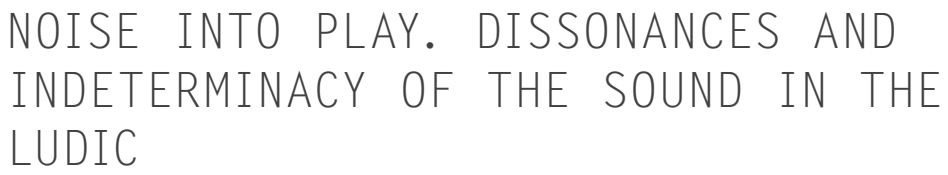

\section{Abstract}

This article aims at presenting a correlation study between the concept of sound noise and the indeterminist aspects of the ludic experimental interaction, focusing on the Survival Horror Games. Based on the ability of sound to cross the display interface and connect to the real space in which the player is found. It create a connection with the concept of ethics developed by Miguel Sicart. The proposal serves both experimental aspects of sound production that are in the game design and its relationship to the field of sound art, and the possibilities of opening up the field of politics and common action through the players experience, from a semantic movement in the concept of horror that can transform the experience of himself in real space.

Keywords: SOUND ART; LUDIC INTERFACES; GAMEART; VIDEOGAMES; INTERACTION

\footnotetext{
Mañas Carbone11, Moisés \& Marina Pastor Aguilar. 2015. "Noise into play: disonancias e indeterminación de 10 sonoro en 10 lúdico". AusArt 3 (2): 22-30. DOI: $10.1387 /$ ausart.15928
}

\section{AUSART}


Estamos asistiendo a un sensible incremento de la alarma social que ha hecho que muchos autores traten de plasmar nuestra conciencia epocal con la denominación de era del terror ${ }^{1}$, cuya mutación, en relación a la anterior postmodernidad, se produce el 9/11. Desde ese día se ha teorizado mucho acerca de la inseguridad económica generada por la crisis del mismo sentido, del pánico inducido en los vestigios de lo cotidiano por los atentados terroristas de cualquier signo, que ha servido como coartada para la instauración de todo tipo de procedimientos de control poblacional, y se han sobreañadido las posibles inseguridades medioambientales, las crisis sanitarias relacionadas con plagas incontrolables, los riesgos vinculados a la alimentación y al despliegue del sistema ciencia tecnología, así como a la posibilidad de la existencia de estados nación desafiantes que puedan producir armas químicas y biológicas de manera descontrolada para un ataque a cualquier forma de poder hegemónico desplegado en cualquier calle de cualquier ciudad del llamado mundo occidental ${ }^{2}$.

La cuestión aquí no es tanto la de analizar si se está incrementando o no el nivel objetivo de riesgos reales, aunque pensamos que muy probablemente sea una consecuencia de la política del escándalo in crescendo que han asumido los medios de comunicación masivos (Gil Calvo 2003), sino la de estudiar cómo se produce esa cualificación experiencial del riesgo y la de considerar de qué mecanismos y dispositivos podemos disponer para afrontar esa omnipresente visibilidad del horror a la que contribuyen los media.

De manera paralela y también desde los medios hemos asistido a un interminable despliegue de las contrafiguras ficcionales del terror y el postciberpunk distópico: hombres lobo, zombies, vampíros, hordas de criaturas caníbales, fantasmas, casas encantadas, posesiones demoniacas, alienígenas hostiles o el colapso total de las infraestructuras sociales anticipadas en una especie de neocultura del Apocalípsis, quizás porque la conciencia cultural tiende a expresarse casi siempre en los patrones del género de ficción. Los videojuegos no han estado exentos de estas invasiones alegóricas y de figuras de la destrucción, a partir del llamado survival horror.

Desde el pionero y mínimal juego Haunted House [Atari, 1982³ para Atari 2600 hasta la actualidad se han sucedido todo tipo de títulos en los que se hace patente esa sensación de indefensión que nos acompaña en lo cotidiano. En la mayor parte de ellos aunque es posible defenderse de los ataques enemigos, las armas suelen ser débiles e improvisadas: palos que causan un daño limitado, trozos de algún objeto cercano y una creciente vulnerabilidad 
de los protagonistas de los distintos juegos, que, además, se suelen desarrollar en un entorno lúgubre, catastrófico y perturbador ${ }^{4}$. Esto provoca que, con frecuencia, sus mecánicas sean las del sigilo y la huida más que las del enfrentamiento y la lucha directa.

En este artículo nos interesa especialmente el elemento sonoro en los juegos del género antedicho dado que pretendemos defender que desde él es posible conformar ciertas alternativas respecto al consentimiento que solemos otorgar a las medidas adoptadas por los poderes fácticos para consolidar una sensación de seguridad en los ciudadanos, y que son medidas que tienden al control de los mismos manteniendo además una actitud de irrupción en espacios de intimidad. Podríamos afirmar con Deleuze en este punto que "en las sociedades de control, lo esencial ya no es una marca ni un número, sino una cifra: la cifra es una contraseña [mot de passe], en tanto que las sociedades disciplinarias están reguladas mediante consignas [mots et ordre], tanto desde el punto de vista de la integración como desde el punto de vista de la resistencia a la integración. El lenguaje numérico de control se compone de cifras que marcan o prohíben el acceso a la información" (Deleuze 1999, 279).

Los sonidos de los videojuegos comunican el espacio virtual de la interface del juego con el espacio real en el que se inserta el jugador. El sonido expande el espacio de juego, invade el espacio propio y es el elemento que pone en comunicación la virtualidad de lo que acontece con el espacio del entorno real. Por ello es susceptible de articularse como un dispositivo que permite también propiciar respuestas en el entorno real desde las que optar, más allá de la aprobación en relación a medidas coercitivas por propuestas más vinculadas a la libertad, viendo la dicotomía entre ambos términos como falsa. Para ello se hacen necesarias las que Miguel Sicart denomina "gameplays éticas", y que son aquelllas que "inducidas desde el juego pueden ser valiosas desde un punto de vista moral" (Sicart 2009, 46), así como usuarios cuya experiencia desborde los principios del puro entretenimiento. Vamos a analizar cómo pueden confluir ambos elementos en la experiencia sonora propiciada por los videojuegos del género "survival horror", y de ciencia ficción de carácter independiente y experimental.

Huizinga ya definió el juego como una actividad libre ejecutada "como si" y sentida como algo situado fuera de la vida corriente. Nos interesa aquí específicamente este "como si" para manifestar cómo es posible ver en esa capacidad del juego para hacer que exista otro orden, otro espacio y otro tiempo, los modos de producir alternativas factibles a esa condición incierta que adopta 
la vida. El juego, gracias a su capacidad de instaurar ese círculo mágico, nos permite articular conductas de ensayo y error, dado que desencadena una actividad que es reversible, es decir, que puede volver a empezar siempre, eliminando los inevitables encadenamientos y consecuencias del sentido lineal del tiempo. Lo mismo sucede con el fenómeno sonoro en relación al cual los videojuegos reactualizan toda la vertiente experimental de lo sonoro puesta en juego por las vanguardias. De este modo, se conjugan lo experiencial que remite a la vida y lo experimental que remite a la producción estética del terror, lo que aquí denominamos horror.

Respecto al ámbito experimental, hay que comenzar anotando que los videojuegos, por su naturaleza interactiva, cuentan con elementos de audio adaptables a las decisiones del jugador las cuales se presentan como formas referenciales y emergentes, partes vivas de la experiencia y que pueden llegar a perturbar el supuesto equilibrio audiovisual, incidiendo directamente en esa descompensación de la experiencia del jugador. Podemos apreciar esto en el juego artístico OSD [19995] de Jodi basado en una modificación (hack) del juego de acción en primera persona Wolfenstein 3D [id Software, 19926] , donde el audio se presenta como un elemento casi arquitectónico que distensiona y cuestiona el juego original y la propia modificación realizada sobre él. No sólo podemos apreciar esta relación en ese ejemplo artístico sino también en desarrollos comerciales como ZombieU [Ubisoft, 2012 ${ }^{7}$ ], donde el entorno a través de la interacción del usuario en el juego se convierte en un experiencia agresiva, límite en términos sonoros, que nos recuerda las experiencias de la vertiente más hardcore punk contemporánea o nos remite, salvando las distancias, a las composiciones tensionadas del estadounidense Morton Subotnick como por ejemplo Silver Apple of the moon (1967).

En los juegos tradicionales hay dos tipos principales de audio dinámico que se producen: el audio interactivo, que responde a las acciones inmediatas de los jugadores, y el audio de adaptación, que responde al propio sistema de juego, ya sea a la reacción o a la predicción de eventos. Además, se encuentra la banda sonora del juego, que cada día es más dinámica y procedural. Lo sonoro se modula de acuerdo con efectos de sonido, diálogos en las que se conjugan tanto elementos diegéticos como extradiégeticos. Existe un territorio compartido y de intersección entre el área de diseño de los videojuegos y las teorías de arte sonoro contemporáneas. Los videojuegos se dedican al mundo del audio en varias formas. Mientras al audio siempre ha sido un componente importante de los videojuegos, en su historia reciente, su sonificación genera 
confluencias con el arte sonoro que llega a extender las fronteras de ambos campos.

En los "survival horror games" algunas de las composiciones suelen estar basadas en sonidos y ambientes industriales que caracterizan un submundo decadente y obsesivo. Usando como base sonidos tomados de diferentes máquinas, sus modificaciones y efectos, crean una atmósfera ruidista y metálica que nos sumerge en una apuesta sonora que bien puede ser remitida al ruidismo de Luigi Russolo pero que también lo desborda, puesto que se centra en un mundo postindustrial, en una distopía tecnológica en la mayor parte de los casos. Se articula así una narrativa electroacústica, una relectura a lo Pierre Henry ([1953] 2011) que nos sorprende al escuchar sonidos que identificamos fácilmente y que se convierten en algo con sentido rítmico e instrumentado: pasos, humedades, chirridos de puertas, intermitencias lumínicas, artefactos mecánicos, entre otros, como es el caso del juego que transcurre en el hospital psiquiátrico de Mount Massive, Outlast ${ }^{8}$ [Red Barrels Games 2013²].

En otros casos se producen auténticas obras de música concreta, al estilo de Pierre Schaeffer, dado que en éste género la acusmática juega un papel muy relevante para tratar de obligar a la audiencia a una escucha activa tendente a poder anticipar la presencia de enemigos y a ingeniárselas con el suficiente tiempo para programar sus reacciones, acusmática porque en ningún momento aparece la fuente de procedencia y la causa del sonido. Desde aquí se articulará el papel de una interacción entre una escucha activa y una inactiva en relación a aquellos sonidos causados por el jugador como en Ju-on The Grudge-Haunted House simulator [Feelplus, 200910]. Así tanto el diseñador como el usuario tienen la oportunidad de jugar con diferentes narraciones sonoras y de articular los tres modos de la escucha definidos por Michel Chion: la semántica, la reducida y la causal. Este relación-esquema se aprecia claramente en la tercera entrega del juego Fatal Frame: The Tormented [Tecmo, 2008 ${ }^{11}$ ] para PS2.

Además la influencia de Cage y Fluxus se deja sentir también en la interacción lúdica que realiza el jugador para producir y remezclar su propio entorno visual y sonoro como podemos apreciar en Memory Broken dimensión [Greenlight, $2014^{12}$ ]. Esta actitud coincide con la que emergió en la década de los 50 del siglo XX en la que el arte sonoro y la interactividad se cruzaron en la obra de Cage, concretamente en sus 4'33"' [1952], ya que se trata de un bloque de tiempo en el que es la audiencia la que emite sonidos, considerados como externos intrusivos y extramusicales en piezas tradicionales. Es el usuario el 
que se convierte en ejecutante y generador del sentido final de los videojuegos. Del mismo modo, en los modos de interacción entre la voz hablada (o el texto) y la música, encontramos los trabajos de Alvin Lucier (2001) o el concepto de paisaje sonoro acuñado por Murray Schafer. Esta aspecto de paisaje sonoro, entendido como capa extrasensible, adaptada al entorno se puede apreciar en el juego independiente para PC Slender the eight pages [Parsec Productions $2012^{13}$ ], el cual plantea una base reiterativa básica de graves de larga duración que recubre todo el espacio a modo de epidermis y que es desgarrada en ocasiones por notas agudas repetitivas y efectos de ruido y desconexión electrónica.

En muchos casos los juegos cortos, tácticos, realizados con una resolución gráfica tosca y valores de producción rudimentarios, los que Alex Galloway denomina "casi antivideojuegos" tratan de algo más que de revestir la imagen alternativa de las convenciones que establece el propio medio. Construir una acción radical, experimental e innovadora en la cultura del videojuego no sólo requiere la creación de algoritmos alternativos (Galloway 2006) sino también gente radical que transforme la experiencia de entretenimiento superficial en una experiencia que puede ser radical activando la imaginación. Es el caso del colectivo Molleindustria o juegos radicales creados por diseñadores independientes como Seven minuts [Virtanen, 2008 ${ }^{14}$ ].

De este modo, si en todo lo que tiene que ver con lo experimental reclamábamos diseños de reglas éticos, que puedan expandir la creatividad hacia el entorno real, y en el caso del arte sonoro incidíamos en el hecho físico evidente que se produce, no podemos obviar que corresponde a los usuarios la actitud activa de trascender esa experimentalidad y saber convertirla en experiencia. Igual que Pierre Schaeffer y su música concreta sacaron partido del sonido de los vinilos rayados que cerraban el surco produciendo un loop sonoro: corresponde a los jugadores aceptar, críticamente o no, las restricciones impuestas por el sistema de juego para obtener conocimiento o entretenimiento, para idear alternativas o conformarse con el mundo tal cual es. Nos referimos a esa vertiente política que el propio Sicart (2009) reclama para rechazar los juegos persuasivamente políticos ya que para él "los verdaderos efectos políticos de estos objetos tienen lugar cuando nos ocupamos de ellos, es decir, cuando se convierten en instrumentos para la expresión política". Jugar es mucho más que simplemente delegar la responsabilidad en los diseñadores de videojuegos. Implica, consciente o inconscientemente, asumir la actitud de Fluxus cuando a través del humor, del juego, de la burla y la provocación subvierte y devasta aquellos contenidos conceptuales que conforman 
la estructura de poder que subyace a los videojuegos. Alex Stockburger (2009) propone una categorización de diferentes estrategias artísticas en el contexto de los videojuegos una de las cuales se remite a las modificaciones realizadas por sus usuarios. En este caso encontramos trabajos de "sonichima" (metamorfosis que juega con el término machinima), palabra acuñado por el artista Julian Oliver (2003) para designar aquellas perturbaciones de la aplicación y el desarrollo de las normas y reglas de juego para desvelar cuales son las funciones subyacentes de su jugabilidad que atañen al sonido. Podemos destacar aquí, por ejemplo, Quake II [id software, 1997], Quilted Thought Organ [Julian Oliver, 1999] que permite al usuario activar efectos de sonido asociados a los objetos en el espacio de juego. De este modo, por ejemplo, las mecánicas asociadas a las colisiones funcionan como un sintetizador sonoro que permite la instrumentalización en vivo como sucede en la experiencia con el clásico cyberpunk System shock 2 [Irrational Games- Looking Glass Studios, 199915] donde la navegación (walkthrough) y colisiones con los diferentes espacios de la nave espacial e interacción con el inventario convierten ese "paseo" en una experiencia de creación sonora en tiempo real paralela pero igual de intensa que los propios objetivos del juego que no son otros que la supervivencia.

Con todo, quizás lo importante es, como afirma Frasca (2011), "poder desarrollar un proceso crítico, aprender las reglas, cuestionarlas y hasta modificarlas $-y$ algunos juegos lo permiten-. Este proceso, que se dio con el teatro y el cine, con los videojuegos recién lo estamos aprendiendo como sociedad". Hay que comenzar a pensar que es posible incorporar y aprovechar la estructura de simulación que ofrecen los videojuegos e incorporarla en todos los ámbitos, y el territorio del sonido es uno de ellos. Los videojuegos no son la solución, sino una herramienta más, y el territorio de lo sonoro uno de los campos de desarrollo de los avances creativos y experimentales de las propuestas artísticas desarrolladas en el siglo XX que se consolidan en este territorio en el XXI. Se trata de acceder a jugadas vinculadas a una activación de la imaginación. Coincidimos con Pajares Tosca en que "La unión de acción y ficción sucede fuera del juego, en la imaginación de los jugadores, espoleada por los ingredientes ficcionales que a la manera de props de teatro recrean el escenario figurado" (Pajares Tosca, 2009).

La bisagra entre la experiencia y la experimentalidad se traduce en la indeterminación, puesto que depende de la experiencia propia que decida desarrollar cada jugador. Cualquier estado particular de un juego es polisémico y abierto y puede no estar tan sometido a las reglas preestablecidas, que pueden ser manipuladas. Más allá de las herramientas sonoras tradicionales, los video- 
juegos pueden funcionar como estrategias cognitivas y dinámicas indeterminadas. Después de todo jugar es luchar contra la eficiencia, la seriedad y el determinismo técnico conceptualmente desarrollados a partir del horror de lo cotidiano, y para ello en los juegos el elemento sonoro es fundamental.

\section{Referencias}

Borradori, Giovanna. 2003. La filosofía en una época de terror: Diálogos con Jürgen Habermas y Jacques Derrida. Traducción de Juan José Botero y Luis Eduardo Hoyos. Madrid: Taurus

Cage, John. 1961. Experimental Music: Doctrine. En Silence: Lectures and Writings. Long Ln, Middletown, CT: Wesleyan University Press

Deleuze, Gilles.1999. Conversaciones 1972-1990. Valencia: Pretextos

Duque Pajuelo, Félix. 2008. Terror tras la postmodernidad. Lecturas de Estética. Madrid: Abada

Frasca, Gonzalo. 2011. "El juego es esencial como territorio de aprendizaje". Declaraciones del artista (texto y videograbación) en el Blog de la Universidad Nacional de Rosario, el 7 de nov. www.unr.edu.ar/noticia/4234/gonzalofrascaquoteljuegoesesencialcomoterritoriodeaprendizajequot

Galloway Alexander R. 2006. Gaming: Essays on algorithmic Culture. Minneapolis, MN: University of Minnesota Press, pp.125-6

Gil Calvo, Enrique. 2003. El miedo es el mensaje: Riesgo, incertidumbre y medios de comunicación. Madrid: Alianza

Henry Pierre. (1953) 2011. "Le voile d'Orphée I", Vídeo de Youtube, 27:00. 8 dic., https://www. youtube.com/watch?v=|AxGbZgzuAU

Huizinga, Johan. (1938) 2007. Homo Ludens. Madrid: Alianza

Lucier, Alvin. 2001. "Alvin Lucier, The official website of the composer Alvin Lucier". Última modificación 11 jun. 2015, acceso 22 oct. 2015. http://alucier.web.wesleyan.edu/

Neuhaus, Max. 2000. "Sound Art?". Prólogo al catálogo de la exposición Volume: Bed of Sound, New York: P.S.1 Contemporary Art Center www.max-neuhaus.info/soundworks/ soundart/SoundArt.htm

Oliver, Julian. 2003. "Diaspora: Experimental Music, 2003 (Synaesthesia 005 release)". En Julian Oliver [web oficial del artista]. Acceso 23 sep. 2015. http://julianoliver.com/output/ tag/sonichima

Pajares Tosca, Susana. 2009. “¿Jugamos una de vampíros? De cómo cuentan historias los videojuegos”. Comunicación 7(1): 80-93

Russolo, Luigi. (1913). 1998. El arte de los ruidos. Cuenca: Centro de Creación Experimental UCLM

Schaffer Murray, Raymond. 2013. El paisaje sonoro y la afinación del mundo. Barcelona: Intermedio

Sicart, Miguel. 2009. "Mundos y sistemas: Entendiendo el diseño de la gameplay ética". Comunicación 7(1): 46 
Stockburger, Axel. 2009. "From Appropiation to Aproximation". En Videogames and art, edited by Andy Clarke and Grethe Mitchell, 25-37. Bristol: Intellect

Subotnick, Morton. (1967) 2011. "Morton Subotnick, Silver apples of the Moon". Vídeo de Youtube, 4:22. 24 marzo, https://youtu.be/EelvKqhu1M4

\section{Notas}

${ }^{1}$ Por citar sólo algunas referencias podemos aludir aquí los diálogos de Giovanna Borrador con Jürgen Habermas y Jacques Derrida (La filosofía en una época de terror Taurus, 2003) a Félix Duque (Terror tras la postmodernidad. Abada, 2008) o los recientes desarrollos del materialismo especulativo que partiendo del universo de los objetos de Alan Badieu y del mundo de los objetos en distintos referentes filosóficos, desarrollan como telón de fondo nihilista el concepto de horror cósmico de Lovecraft a partir de productos de la producción cultural más underground como cómics, fanzines, el black metal en música, etc. Todos ellos son considerados productos culturales que han explorado lo macabro cósmico.

${ }^{2}$ En el momento de escribir este artículo se han producido una serie de atentados terroristas en Paris. Aunque es demasiado pronto para hacer una alusión a los mismos, puesto que aún se desconocen las consecuencias, e incluso qué, cómo y por qué se han producido éstos, no podemos evitar hacer una alusión a la imposición de un estado de sitio en la ciudad, desde el llamado "estado de emergencia", así como al hecho de que ha sido el "sonido" del himno francés, La Marsellesa, lo que se ha convertido en icono de los hechos. La versión sonora de los mismos también se ha convertido en un recurso repetido mediáticamente por primera vez en sucesos de este calado.

${ }^{3}$ A partir de este momento incluiremos en las referencias a videojuegos en las notas al final gameplays de los mismos para facilitar su audición. Haunted House (gameplay) https:// youtu.be/D7u7xROfsaQ

${ }^{4}$ Después de todo, la mayoría de los monstruos enemigos son seres que ya han muerto: zombis, fantasmas, momias... o seres que no pueden morir, como los vampiros.

${ }^{5}$ OSD (gameplay) https://youtu.be/24KQiyOU Uk

${ }^{6}$ Wolfenstein 3D (gameplay) https://www.youtube.com/watch?v=NdcnQISuF Y

${ }^{7}$ ZombiU (gameplay) https://youtu.be/BicJFsE8rl

${ }^{8}$ Outlast (trailer) https://youtu.be/2GPf3MdVOKI

${ }^{9}$ Red Barrels Games http://www.redbarrelsgames.com/

${ }^{10}$ Juon: The grudge haunted House simulator (gameplay) https://youtu.be/e3 2IGiB9DQ

${ }^{11}$ Fatal Frame: The Tormented (gameplay) https://youtu.be/3aTxUqXNTxk

${ }^{12}$ Memory of a Broken Dimension http://www.brokendimension.com/

${ }^{13}$ Slender: The Eight Pages http://www.parsecproductions.net/slender/

${ }^{14}$ Seven minuts (gameplay) http://koti.mbnet.fi/erkkavir/sevenminutes.php ; https://youtu.be/ N9nY750fZ3M

${ }^{15}$ System shock 2 (gameplay) https://youtu.be/8kUT6XzRvhc 